\title{
Comunidades virtuales (fandoms) de videojuegos y su interacción: League of Legends Ecuador
}

\section{Virtual communities (fandoms) of videogames and their interaction: League of Legends Ecuador}

Kevin Ruíz Semillero Mitos Shinhwa Machala - Ecuador kpruiz_est@utmachala.edu.ec

Laddy Quezada Tello Semillero Mitos Shinhwa Machala - Ecuador lquezada@utmachala.edu.ec

Gabriel Iñiguez Parra

Semillero Mitos Shinhwa Machala - Ecuador giniguez@utmachala.edu.ec

Revista Cumbres Vol.5 №1

Versión impresa ISSN 1390-9541

Versión electrónica ISSN 1390-3365

http://investigacion.utmachala.edu.ec/revistas/index.php/Cumbres 


\title{
RESUMEN
}

Actualmente en la sociedad se observan transformaciones dentro de los procesos sociales como consecuencia de la influencia de las nuevas tecnologías en los modos de vida modos de vida de las diversas generaciones, provocando que estos se adapten a plataformas digitales atemporales, donde no existe las distancias ni horarios que los separen de sus similares conectados a la red desde otros puntos del mundo. Esto a su vez genera nuevas formas de ocio en los millenials, los que día a día usan los videojuegos y en algunos casos los convertirse en gamers profesionales reconocidos, como el caso de los participantes de League of Legends. En esta investigación se caracteriza el perfil de los jugadores pertenecientes a la comunidad de League of Legends Ecuador desde la entrevista a expertos; así mismo desde un estudio de etnografía virtual se trata de identificar los canales de comunicación 2.0 que estos utilizan para su interacción mientras se clasifican los contenidos transmedia producidos desde la marca dueña del videojuego hacia fans y los generados desde los gamers para sus semejantes.

Palabras clave: Fandoms, narrativa transmedia, gamers, prosumidor.

\begin{abstract}
Currently in the society transformations within social processes are observed as a result of the influence of new technologies on lifestyles modes of life of different generations, causing them to adapt to timeless digital platforms, where distances do not exist nor schedules that separate them from their peers connected to the website from other parts of the world. This in turn generates new forms of entertainment in the millennials, which day by day use video games and in some cases become recognized professional gamers, as in the case of League of Legends participants. In this research the profile of the players belonging to the League of Legends Ecuador community is characterized from the interview with experts; Likewise, from a study of virtual ethnography, it is about identifying the 2.0 communication channels that they use for their interaction while classifying the transmedia contents produced from the brand that owns the game to fans and those generated from the gamers for their peers.
\end{abstract}

Keywords: Fandoms, narrativa transmedia, gamers, prosumidor.

\section{Cumbres}




\section{INTRODUCCIÓN}

La evolución de una sociedad cada vez más inmersa en una cultura tecnológica ha provocado el nacimiento de nuevas formas de comunicación, cada vez más arraigadas a una dinámica bilateral, en donde los consumidores toman una voz activa en los procesos de creación de la información que hay a su alrededor, tal es el caso de los videojuegos, que luego de un proceso de transformación y adaptación a las necesidades de los usuarios presentan contenidos nuevos que generan expectativa en los consumidores quienes mantienen un alto interés por adquirirlos.

Por ello, según Charsky (2010), los videojuegos son una herramienta que podemos volver a redimensionar como elemento didáctico, pero también podemos aislar la estructura comunicacional formulada para presentar sus productos y convertir a los usuarios en prosumidores ${ }^{1}$. Tal como es el caso de League of Legends, que ha logrado captar la atención de gran parte de la población global llegando a niveles competitivos, pero una de las razones más notable de su éxito es la presentación de sus narrativas transmediales, donde la interacción de los usuarios ha logrado que en muchos casos generan contenidos de autoría de cada usuario.

Todo este fenómeno demuestra una transformación en la realidad, poniendo como prioridad a la tecnología, la que debe responder a la responsabilidad de la interacción social de las nuevas generaciones, como lo afirma Alonso(2008) "de forma natural, las imágenes ponen en marcha procesos que desempeñan un papel fundamental en el desarrollo de la comprensión del mundo social en los niños", pues los espacios abiertos en donde se compartían momentos de diversión entre amigos del barrio han ido desapareciendo, dejando como únicos medios de interacción a las tecnologías de la comunicación.

\section{Objetivo General}

Estudio del perfil de los jugadores de League of Legends, comunidad Ecuador e identificación de los canales de comunicación 2.0 utilizados para la interacción y clasifican los contenidos transmedia producidos desde la marca frente a los generados por los gamers.

\section{Objetivos específicos}

- Estudio del perfil de los jugadores de League of Legends, comunidad Ecuador.

\footnotetext{
${ }^{1}$ Prosumidor: Se refiere a las personas que consumen productos comunicacionales y en base a su experiencia o deseo genera nuevos contenidos para medios digitales en su mayoría: Un caso relevante son los youtubers. Videos o historias que se pueden visualizar en la net y no pertenecen a la marca oficial del producto sino que es una adaptación hecha por los fans. Productor + Consumidor $=$ Prosumidor
} 
- Identificación de los canales de comunicación 2.0 utilizados para la interacción de miembros del fandom.

- Clasificación de narrativas transmedia producidos desde la marca y los generados por los gamers.

Cada generación ha desarrollado características propias que las diferencia las unas de las otras como es el caso de los millenials, quienes nacieron en una era llena de nuevas tecnologías, fueron los que recibieron el peso total del desarrollo. Y es por esto que aspiran a mantenerse en estas tecnologías, por lo que es común ver a los jóvenes invirtiendo de su tiempo en videojuegos que hoy en día se han convertido en un nuevo mercado viendo la necesidad de investigar el contexto de estos productos tecnológicos, que poco a poco se vuelven parte de la cultura popular, por eso se deben sintetizar estos modelos comunicacionales para aplicarlos en diferentes áreas de la comunicación de marca.

Debido a esto, en Ecuador existe un aumento de interés por parte de la población adolescente y adulta en productos de entretenimiento como videojuegos instalados en sus computadores o consolas que han transformado las necesidades sociales y comunicacionales dentro de los miembros de la sociedad al dedicar mucho de su tiempo libre a jugar, siendo el caso más visibles el de League of legends, que según la página oficial de su desarrolladora Riot Games (2016) la define así:

Es un juego competitivo en línea de ritmo frenético, que fusiona la velocidad y la intensidad de la estrategia en tiempo real (ETR) con elementos de juegos de rol. Dos equipos de poderosos campeones, cada uno con un diseño y estilo de juegos únicos, compiten cara a cara a través de diversos campos de batalla y modos de juego. Con un plantel de campeones en constante expansión, actualizaciones frecuentes y un emocionante panorama competitivo, League of Legends ofrece posibilidades de juego ilimitadas a usuarios de todos los niveles de habilidad.

Ante esto se hace el presente estudio acerca de las Comunidades virtuales (fandoms) de videojuegos y su interacción: League of Legends Ecuador.

\section{MATERIALES Y MÉTODOS}

\section{Transmedia}

Bajo sus casi infinitas formas, el relato está presente en todas las épocas, en todos los lugares, en todas las sociedades; el relato empieza con la historia misma de la humanidad; no hay, nunca ha habido, un pueblo sin relato (Barthes, 1977: 2).

Para Scolari (2015), la emergencia de la World Wide Web constituyó un verdadero terremoto. La web no es un medio más como la televisión o la radio: es un gran nicho dentro del ecosistema de medios que, desde su aparición, no paró de generar nuevas formas disruptivas de comunicación...es un espacio abierto a la innovación donde se generan nuevos entornos y experiencias comunicativas. 
En la investigación realizada por Scolari (2017) indica que Henry Jenkins fue el primero en emplear este término publicado en Technology Review en el año 2003 en el que afirma que "hemos entrado en una nueva era de convergencia de medios que vuelve inevitable el flujo de contenidos a través de múltiples canales".

No obstante, durante el desarrollo de nuevas realidades tecnológicas más útil se convierte este concepto. De hecho, la narrativa transmedial y los fandom de las diferentes propuestas multimedia en videojuegos son la base estructural de los estudios de varios investigadores. Debido a esto, Crisóstomo (2016) dibuja el ambiente de la influencia que tienen los seguidores basado en los elementos constructivos y al ámbito de la narrativa transmedia.

No se debe olvidar que un medio es un transporte, que, en lo transmedial va a dirigir al usuario a un mundo narrativo que ofrece diferentes novedades, por eso es importante tomar en cuenta el timing o planificación temporal para elaborar las tramas narrativas, que para Costa (2012) se debe tener establecido los momentos en el que los contenidos transmediáticos se ejecutarán en las distintas plataformas, para que cumplan con la finalidad con la que se crearon, esto influirá en la experiencia del usuario y el grado de participación que tendrán en cada una de ellas.

Pero el verdadero valor de una narrativa son sus contenidos, que basados en lo que nos explica Costa (2012) quien describe a las personas como seres hambrientos de historias, por lo que es indispensable crear una realidad totalmente original que permite sumergirnos en ellas y vivirlas, entonces podemos afirmar que un storytelling es un contenido narrativo diseñado para que el usuario se vea dentro de la historia y que sea capaz de encontrar los detalles para tratar de suponer lo que podría venir.

Como explica Costa (2012) en muchos de los casos de transmedia storytelling que han funcionado, su éxito se debe principalmente a que los consumidores de esta historia se han podido identificar en alguno de los personajes que se describe durante la narrativa, llegando a sentirse muy relacionados emocionalmente hacia estos.

\section{Iconosfera}

Según Ávila (2016) la iconosfera no se limita al proceso superficial de lo que se percibe de un objeto a través de los ojos, sino que es un conjunto de procesos cognitivos de raíz escópica que establece una conexión entre lo subjetivo y la herencia cultural del entorno de un individuo hasta llegar a una percepción de la realidad por encima de lo que divisa.

Sola (2015) afirma que la imaginación y los imaginarios son piezas fundamentales para la cimentación de las bases para estudiar al entorno social y sus derivados (cultura y todo tipo de fenómenos de la comunicación mediática), por lo tanto se puede simplificar a la iconosfera como el conjunto de credos que tienen en común y comparten las sociedades. 


\section{Prosumidor}

El término prosumidor lleva una historia muy amplia en la historia de la sociedad, como Carrero(2012) resalta, la importancia de las dimensiones de este concepto parte en sus orígenes, en los años 70 del siglo pasado, relacionado con otras áreas sociales y productivas de la época, hasta la edad moderna, en donde los recursos digitales han construido un ambiente adecuado para la retroalimentación.

Un prosumidor en los términos de Carrero(2012), "Tiene su origen en la unión de dos conceptos: productor y consumidor" y se catalogan a aquellos individuos que toman parte de un proceso comunicacional bidireccional y se convierten en un medio de comunicación, en donde tienen un papel activo en la creación de contenidos, que en muchos de los casos se proyectan en un futuro producto como es el caso de los videojuegos.

Scolari (2017) manifiesta que las narrativas también se caracterizan por otro componente: una parte de los lectores no se limita a consumir el producto cultural sino que se propone ampliar su mundo narrativo con nuevas piezas textuales. Una narrativa transmedia puede ser sintetizada a través de una fórmula:

$\mathrm{NT}=\mathrm{IM}+\mathrm{CGU}$

NT: narrativa transmedia, IM: industria de medios, CGU: contenidos generados por usuarios.

Puede ser comparado a:

NT= canon (empresa) + fandom (prosumidor)

Si sumamos canon y fandom nos encontramos con mundos narrativos que se expanden a través de múltiples medios y plataformas con la complicidad de sus fans. Estas narrativas se saben dónde comienzan pero nunca dónde acaban.

\section{Propagabilidad}

La propagabilidad se ejecuta luego de una serie de acciones que permiten convertir a un contenido en viral. Para Hernández (2017), aquellos consumidores que se han identificado mucho con un producto, tendrán un nivel de compromiso muy alto y pasarán a convertirse en distribuidores, apropiándose de la identidad para llevar el mensaje hacia demás sujetos, este criterio lo refuerza Herrero (2017) desde la relación que debe existir entre los medios masivos y los públicos para construir una participación democrática y responsable. Aportando al concepto de libertad, las sociedades que formulen sus contenidos a través de medios de comunicación.

En conclusión, se puede afirmar basado en la opinión de estos autores, que la propagabilidad nace a partir de la necesidad de expresar libremente los contenidos construidos por los usuarios, y que aunque con la modernidad a través del internet se ha globalizado, los procesos virales han venido desarrollándose desde hace mucho tiempo atrás. Esto implica el papel fundamental de los consumidores, que no solo establecen el patrón de uso, sino que establecen las tendencias que nacen a partir de las masas. 
En este sentido Guerrero (2016) indica que la expansión de contenidos transmedia gestionada desde arriba (top-down) por los productores se complementa con las expansiones desde abajo (bottom-up) realizadas por los usuarios y difundidas en plataformas colaborativas de fan fiction, se las suele denominar contenidos generados por los usuarios (CGU).

\section{Fandoms}

Según (Camarero, 2017) los seres humanos formamos redes sociales desde el principio de los tiempos. Sin estas no hubiera sido posible evolucionar como raza y sociedad. Dichas redes sociales tienen lugar en "comunidades tradicionales” y, por lo tanto, se las considera analógicas. Esto es porque tienen lugar en la presencialidad. Las redes sociales pueden ser analógicas (cara a cara) o digitales (a través de un ordenador o dispositivo móvil)(Camarero-Cano, 2015)

La aparición de nuevas tecnologías ha permitido el acercamiento de muchos contenidos a usuarios que aprecian estos, sin embargo el término de fandom ha generado un fenómeno que impactaría a una sociedad en crecimiento, porque aunque son prosumidores se van apartando a un nivel superior y esto según Crisóstomo(2016):

"Se encuentra en estrecho apego a que encuentran una identificación personal en cada una de las historias que va más allá del tiempo llegando incluso a influir en su esencia como ser humano, haciendo el centro de su vida las narrativas que se consumen, por eso es que esta población es susceptible a la información que los media presentan, llegando a convertirse en un prototipo de cómo podrían llegar a funcionar los medios masivos."

Scolari (2017) indica que la actividad de los fans conforma lógica de la apropiación, el remix y el mashup. La actividad de los fans conforma un extenso territorio muy difícil de mapear, está impulsada por la pasión y no tiene límites narrativos ni temporales. Las personas interaccionan de manera multidireccional y pasan a ser "webatores" (Pisani y Piotet, 2013); es decir de ser ciudadanos pasivos se convierten en sujetos activos, protagonistas en el acto de comunicar.

En las manifestaciones de contenidos transmedia generados por los usuarios se pueden encontrar las siguientes tipologías: Fan fiction (fanfic o fic): narraciones en formato de texto, Fan vid: vídeos de corta duración y películas realizadas a partir de imágenes y músicas de una tercera fuente o propia. Fan art: dibujo, pintura y arte gráfico. Machinima: vídeos o películas realizados manipulando el motor gráfico de un videojuego en 3D. (Guerrero, 2016).

Las creaciones de fans "se representan generalmente como complemento al enriquecimiento de la imaginación y escapismos propios de los mundos de fantasía" (Grandío, 2015) 


\section{Glamificación}

El desarrollo de la sociedad va en dependencia de los conocimientos que han adquirido en su historia: experimentos, investigaciones y más trabajos que han permitido encontrar información valiosa para la humanidad, pero en el contexto actual de las nuevas tecnologías se presenta un dilema, la aplicación de estrategias pedagógicas clásicas deben adaptarse a esta nueva población hiperinformada, Scolari(2013) genera varias interrogantes dirigidas a los individuos que interactúan en los videojuegos: ¿Por qué dedicar largas jornadas sentados frente a un ordenador? ¿Qué los motiva a realizar arduas investigaciones con tal de mantenerse informado de nuevas estrategias para poder destacar en estos videojuegos?, pero su argumentación ante esta problemática plantea una transformación de la ideología pedagógica, diciendo que el cambio no debe ser tecnológico sino conceptual.

Por esto dentro estos procesos de enseñanzas se deben identificar los elementos inmersivos que destacan en estas nuevas tecnologías para ser empleadas en la construcción del conocimiento, como menciona Rose(Rose, 2011) "llevan a profundizar tan hondo como se quiera sobre algo que nos interese"

\section{Video juegos masivos}

La evolución en esta era tecnológica, ha lanzado a la sociedad a un mundo ilimitado de oportunidades en varios aspectos, el acceso a internet ha dado el nacimiento a diferentes comunidades a través de los videojuegos sociales, cooperativos y competitivos, por esto, basados en Bertrán(2016) a esta clase de juegos en línea se les denomina MMO (Massively Multiplayer Online) que dependiendo de su modo de juego pueden ser MOBA o MMORPG, que en la actualidad han sumado millones de seguidores que buscan compartir o destacar con sus habilidades entre esta población.

\section{MMORPG}

Los Massively Multiplayer Online Role Playing Games (juegos de rol online) para Kahn(2015) permite a los usuarios disponer de un avatar que personalizan y con el que realizarán batallas en las que tendrán varias recompensas que le permitirán aumentar su nivel y con ello sus habilidades.

Por lo tanto, la inmersión en comunidades virtuales de este tipo es de suma importancia para mantener a los jugadores en constante conexión, pues deben en varias ocasiones unirse para conquistar misiones e investigar los territorios que ofrecen estos juegos.

\section{Moba}

El objetivo principal de los MOBA a criterio de Nuangjumnonga(2012) es "destruir la base del equipo contrario que es la estructura principal que 
está fuertemente custodiada en los lados opuestos", en esta competición se destaca la sinergia de habilidades de los personajes del videojuego como de los jugadores quienes son responsables de su victoria o derrota según la decisión que se tome en el transcurso de la partida.

Aunque dispones de un personaje que acompañará en la partida, es mucho más rápida que un MMORPG, pues se debe escalar las habilidades más rápido que el enemigo, para tomar ventaja en la batalla, coincidiendo con Nuangjumnonga(2012) en la importancia del oro obtenido para plantear una mejor estrategia que la del enemigo.

\section{METODOLOGÍA}

Para la elaboración de este trabajo, se establece una metodología cuanti-cualitativa, que comprende la aplicación de análisis de observación (etnografía virtual), a partir de la generación de una tabla de variables se analizará al canal de la comunidad League Of Legends Lan Ecuador, los contenidos que se publican y los niveles de interacción entre los miembros de la comunidad.

También se realizarán entrevistas a especialistas en diferentes ramas del conocimiento para caracterizar los perfiles de los jugadores desde una perspectiva multidisciplinaria que permita abordar de mejor manera el tema de investigación.

Es necesario entender el contexto en el que se desarrolla la investigación, por lo que se aplicarán encuestas en la comunidad de League of Legends Ecuador, para examinar la realidad de la población que consume este videojuego, donde podremos medir cuantitativamente sus respuestas mediante la identificación de los usuarios más activos, obteniendo una valoración real de su experiencia. Las técnicas empleadas para la recolección de información en esta investigación son las siguientes:

- Revisión bibliográfica

- Etnografía Virtual

- Entrevistas

- Encuestas

A continuación, los principales resultados obtenidos en la aplicación de las diversas herramientas de investigación.

\section{RESULTADOS}

\section{Perfiles de los jugadores online}

Según Del Moral(2017), las personas que consumen estos productos de entretenimiento online, son aquellas que han logrado crear un vínculo con éstas y la principal razón de su fidelización es la participación activa dentro de estos contextos desempeñando una función específica, que llevan al usuario a permanecer en constante atención para poder exteriorizar sus habilidades y alcanzar los objetivos para la victoria en una partida. 
En la presente investigación se ha aplicado la técnica de entrevistas a diferentes profesionales en diversas ramas convergentes para la investigación (Psicólogo, docente, especialista en tecnología, jugador semiprofesional y streamer) con la finalidad de caracterizar el perfil de los jugadores del fandom League of Legends en diferentes aspectos lo que permitió la realización de la siguiente matriz:

Tabla 1. Conceptualización multidisciplinaria de variables por parte de profesionales

\begin{tabular}{|c|c|}
\hline Tema & Idea \\
\hline Cultural & $\begin{array}{l}\text { Las personas buscan diferentes maneras de ocupar su } \\
\text { tiempo y mente debido a diferentes razones. } \\
\text { comunidad. } \\
\text { Conocimiento compartido entre los miembros de la } \\
\text { to de las nuevas generaciones. } \\
\text { contenidos que alimentan su comunidad. }\end{array}$ \\
\hline Comunicación & $\begin{array}{l}\text { Muy poco uso de páginas oficiales, por lo que se } \\
\text { informan mediante videos en canales de Youtube o durante los } \\
\text { streams. } \\
\text { es buena en un } 90 \% \text {, porque existen personas "tóxicas" que } \\
\text { contaminan el juego de los demás. } \\
\text { bersonas dedicadas a videojuegos pueden tener pro- } \\
\text { blemas para relacionarse en la vida cotidiana, sin embargo en su } \\
\text { mundo digital son totalmente opuestos. }\end{array}$ \\
\hline Social & $\begin{array}{l}\text { Pueden reforzar los atributos positivos o negativos de } \\
\text { la personalidad en dependencia del orden de prioridad que le } \\
\text { den. } \\
\text { fuente de ingresos con el que se pueda vivir. } \\
\text { implica una evolución en base a las necesidades sociales. }\end{array}$ \\
\hline Tecnológico & $\begin{array}{l}\text { - Estudios sobre las nuevas tecnologías y videojuegos } \\
\text { han encontrado los conceptos de Gamificación y Juegos serios, } \\
\text { que contribuyen al proceso educativo de los estudiantes. } \\
\text { enseñanza como la Gamificación. } \\
\text { endodogías en el proceso de } \\
\text { el rendimiento físico de estas actividades. }\end{array}$ \\
\hline
\end{tabular}

Además de esto se contrasta con entrevistas a jugadores orenses de nivel semiprofesional y amateur de la League of Legends, obteniendo lo siguiente:

Según entrevista a Jovanny Coronel (2018) jugador semiprofesional, indica en nuestro país el tema de los videojuegos es mal vista por la población en base al imaginario que consideran esta práctica como un vicio en vez de un deporte. Además, puede significar problemas familiares a causa del tiempo que deben invertir para la profesionalización del juego, aproximadamente 12 horas al día con la finalidad de ser un "PRO".

En este sentido es conocido que League of Legends es uno de los e-sports (juegos electrónicos) más conocidos, en el extranjero hay universidades que enseñan específicamente las modalidades del juego y la expansión de LOL 
por medio del trabajo de su creadora RIOT ha alcanzado gran cantidad de participantes incluso en la celebración de su torneo mundial donde delegaciones de varios países como: Korea del Sur, Estados Unidos, China, Alemania, etc. En el 2016, en las competencias hubo 15 millones de usuarios concurrentes viendo las partidas; esto permitió retransmisiones en cerca de 18 idiomas, utilizando 18 equipos de retrasmisión para la cobertura del evento.

Referente al comportamiento de los gamers se encuentra en los testimonios de Julio Rivadeneira y Fabian Fiallo (2018), jugadores amateurs de LOL la coincidencia del hecho que todo depende de los valores o disciplina con lo que cada persona ha sido educada, por ejemplo: hay miles de personas que juegan Shooters pero no por el hecho de jugarlo van a ir a una escuela para disparar a la gente. Este tipo de juego les ha permitido a los gamers incrementar su agilidad mental por el hecho de la toma rápida de decisiones que no afecten al grupo mediante el fortalecimiento del liderazgo, la disminución de las reacciones violentas mediante las amonestaciones correctivas de este Moba para no desvirtuar la realidad de que esto no es más que un juego y permitir la generación de amistades a través de la convivencia digital que luego evolucione a relaciones en plano real.

En este sentido se hace una encuesta dentro del fandom de LOL Ecuador para establecer el tiempo invertido por ellos en el juego, teniendo como resultados la respuesta de 165 participantes activos en la tercera semana del mes de julio 2018. Los datos se organizan en la siguiente tabla:

Tabla 2. Tiempo invertido en el juego

\begin{tabular}{|l|c|}
\hline De 4 horas en adelante & 84 \\
\hline De 2 a 4 horas & 47 \\
\hline De 1 a 2 horas & 34 \\
\hline Total de encuestados & 165 \\
\hline
\end{tabular}

De un total de 165 miembros de la comunidad en facebook de Lol Lan Ecuador que respondieron esta pregunta, el 50.9\% juegan más de 4 horas, un 28.5\% dedican de 2 a 4 horas y un $20.6 \%$ de 1 a 2 horas durante el día. Este promedio confirma lo que los gamers entrevistados manifestaban al hecho que se puede llegar hasta 12 horas entre juegos y entrenamiento para formación de estrategias para vencer en los combates.

\section{Canales 2.0 aplicados por fandom LOL Ecuador}

Coronel (2018), indica que LOL permite el chat de voz para mantener contacto en tiempo real con los miembros del equipo que forman parte de la partida, esto lo diferencia de otros videojuegos que tienen comunicación por voz incluso con quienes no conforman el grupo y aumenta las posibilidades de toxicidad o malos comentarios por parte de diversos gamers. Además, esto evita que las estrategias de equipo sean conocidos por los contrincantes.

En otro caso, los canales de comunicación 2.0 utilizados entre los gamers miembros del fandom LOL Ecuador son de interés para estudiar la interac- 
ción que mantienen en espacios virtuales y la posibilidad de desplazar esta experiencia al intercambio interpersonal. En este sentido encontramos la primera estadística que ubica a la red Facebook como el principal canal aplicado en el proceso de comunicación virtual, seguido por un grupo de whatsapp a través del cual comparten información relacionada a LOL, eventos gamers, reuniones y han surgido espacios para hacer amistades según lo manifiestan los jugadores entrevistados como el caso del nuevo Skype llamado Discord.

Tabla 3. Canales de comunicación aplicados por la comunidad

\begin{tabular}{|l|r|}
\hline Facebook & 82 \\
\hline Foros & 11 \\
\hline Whatsapp & 72 \\
\hline Otros & 0 \\
\hline Total encuestados & 165 \\
\hline
\end{tabular}

De un total de 165 miembros de la comunidad en facebook de Lol Lan Ecuador que respondieron esta pregunta, el 49.7\% emplean Facebook como medio digital de comunicación, un $43.6 \%$ usan Whatsapp y un 6.7\% revisan foros para informarse y estar en contacto con la comunidad de League of Legends.

\section{Clasificación de las narrativas transmedia producidos desde la fuente y los generados por los gamers (fans prosumidores)}

Basado en la etnografía virtual se genera el seguimiento a las interacciones del fandom donde se encuentra que la información parte de la página oficial de League of Legends gestionada por RIOT, en donde la gente comenta e incluso genera contenidos propios desde el gamer para el beneficio de la comunidad virtual; despuntando los "figura LOL" que son aquellos personajes que toman la responsabilidad de acompañar a los demás jugadores para que mejoren su nivel y generando videos clave con trucos o estrategias para subir el nivel en la competencia.

Un caso de los contenidos que la web oficial lanza para los gamers es Nunu, un niño con rasgos indígenas, esto demuestra que RIOT está tomando más en cuenta la región de Latinoamérica por el incremento de fans y cuando se creó el servidor para esta zona apareció el personaje llamado Morgana al que se le creó la skin basado en "La Llorona", una historia tradicional que pertenece a la geografía latina. (Coronel, 2018).

Dentro del presente estudio también se hace visible que una figura LOL local fue "Prodigy" nickname de Jovanny Coronel, mientras jugaba de manera continua en el nivel semiprofesional de este famoso e-sport tuvo hasta 2000 seguidores locales que estaban pendientes a las publicaciones en su fanpage que destacaban por los turcos, estrategias de juego producidas por este personaje.

Además, se obtiene dentro de la revisión de contenidos publicados en el fandom, los de mayor interés por los gamer se refieren al 50.3\% ver memes dentro de su contenido en la comunidad, el $42.4 \%$ publicaciones sobre noti- 
cias, el $6.1 \%$ prefiere ver tutoriales sobre el juego y el $0.6 \%$ videos de jugadas junto al otro $0.6 \%$ prefiere ver los streaming de los miembros de la página.

Tabla 4. Tipo de contenidos preferidos dentro de la comunidad LoL Lan Ecuador

\begin{tabular}{|l|c|}
\hline Memes & 83 \\
\hline Tutoriales & 10 \\
\hline Noticias & 70 \\
\hline Streaming & 1 \\
\hline Videos de Jugadas & 1 \\
\hline Total encuestados & 165 \\
\hline
\end{tabular}

Para establecer los resultados según contenidos publicados en el fanpage de LOL Ecuador durante la tercera semana de julio se encuentra que existen un total de 176 reacciones entre los diferentes contenidos posteados por los miembros del fandom; teniendo mayor número los relacionados a Likes, luego los Me Divierte y en tercer lugar los Me Encanta, esto permite conocer el nivel de identificación que tienen los fans con la información que circula dentro del grupo.

Tabla 5. Reacciones de los fans en redes sociales

\begin{tabular}{|l|c|c|c|c|c|c|c|}
\hline $\begin{array}{l}\text { TIPOS } \\
\text { DE } \\
\text { POST }\end{array}$ & ABREV & $\begin{array}{l}\text { ME } \\
\text { GUSTA }\end{array}$ & $\begin{array}{l}\text { ME EN- } \\
\text { CANTA }\end{array}$ & $\begin{array}{l}\text { ME DI- } \\
\text { VIERTE }\end{array}$ & $\begin{array}{l}\text { ME } \\
\text { ASOM- } \\
\text { BRA }\end{array}$ & $\begin{array}{l}\text { ME EN- } \\
\text { TRIS- } \\
\text { TECE }\end{array}$ & $\begin{array}{l}\text { ME } \\
\text { ENO- } \\
\text { JA }\end{array}$ \\
\hline $\begin{array}{l}\text { Inte- } \\
\text { racción } \\
\text { directa }\end{array}$ & ID & 66 & 6 & 34 & 1 & 4 & 5 \\
\hline $\begin{array}{l}\text { Branded } \\
\text { Content }\end{array}$ & BC & 6 & 1 & 0 & 0 & 0 & 0 \\
\hline $\begin{array}{l}\text { Juegos en } \\
\text { Facebook }\end{array}$ & JF & 0 & 1 & 0 & 0 & 0 & 1 \\
\hline $\begin{array}{l}\text { Conteni- } \\
\text { do promo- } \\
\text { cional }\end{array}$ & P & 25 & 4 & 1 & 2 & 0 & 0 \\
\hline $\begin{array}{l}\text { Conteni- } \\
\text { do infor- } \\
\text { mativo }\end{array}$ & INF & 2 & 0 & 0 & 0 & 0 & 0 \\
\hline $\begin{array}{l}\text { Conteni- } \\
\text { do multi- } \\
\text { media }\end{array}$ & MUL & 12 & 0 & 1 & 1 & 1 & 2 \\
\hline TOTAL & & 111 & 12 & 36 & 4 & 5 & 8 \\
\hline
\end{tabular}

De un total de 165 miembros de la comunidad en facebook de Lol Lan Ecuador que respondieron esta pregunta, el 58.2\% ha generado comentarios, un $25.5 \%$ genera otro tipo de contenidos como stream, publicidad de sus equipos, publicidad de torneos a realizarse; mientras el $16.4 \%$ restante crea contenidos en videos. Los comentarios creados por los usuarios de la comunidad de Lol Lan Ecuador han sido los contenidos de mayor generación en comparación a los videos y a otros contenidos como streaming, publicidades y demás recursos. 
Tabla 6. Contenido generado por los miembros de la comunidad

\begin{tabular}{|l|c|}
\hline Comentarios & 96 \\
\hline Videos & 27 \\
\hline Memes & 0 \\
\hline Otros & 42 \\
\hline Total encuestados & 165 \\
\hline
\end{tabular}

Esto también es validado por los jugadores de LOL que fueron entrevistados para este tema cuando indican que la mayoría de los gamers interaccionan con sus similares a través de su opinión en los post que son colgados dentro del fandom, los principales que se dedican a generar videos con instrucciones son los denominados "figura" y para ello requieren usar software que permita la grabación de pantallas para llevar el curso de cada partida jugada. La óptima calidad de internet no la brindan todos los proveedores por lo tanto a nivel nacional es preferido contratar los servicios de "Celerity - Punto net" e incluso uno de los premios normalmente ofrecido para esta comunidad se refiere a planes de fibra óptica para los ganadores por el lapso de un año, esto lo manifiesta Jhon Bravo (2018) gamer machaleño amateur de LOL.

A esto se suma la publicación efectuada en octubre 2018 por la página de www.sandvine.com en su reporte mundial de video juegos informa que League of legends tiene el $26.42 \%$ del global Gaming traffic share, el Fortnite lleva un $14.85 \%$ y el tercer lugar es Overwatch con el $12.05 \%$. El mismo reporte manifiesta que este juego tiene el $51.53 \%$ de conexiones de juego entre el top de los video juegos; para finalizar el presente documento se generan las conclusiones de investigación a partir del tránsito por los autores teóricos, entrevistas, análisis de contenidos publicados y reportes de fuentes confiables.

\section{CONCLUSIONES}

- A nivel local falta hacer mayor hincapié en el estudio, difusión y preparación para gamers al nivel que lo hacen países con mayor experiencia en las Batallas de este tipo de juego MOBA para educar en salud a todos los usuarios, disminuyendo posibles afecciones como el sobrepeso, diabetes, etc.

- Existe cierto sesgo acerca de este e-sport basado en el imaginario colectivo de que los gamers son personas socialmente retraídas, que es un vicio o incluso que es solo para "vagos" que pierden el tiempo al pasar horas entrenando. Además, se toma como una actividad inmadura e irresponsable en los gamers adultos.

- Parte de la actual formación de la identidad juvenil se basa en el sustancial apego a las tecnologías en donde las nuevas generaciones se expresan e interactúan pero en otro porcentaje de la población sin madurez, esto genera un desapego de la realidad acarreando actitudes tóxicas y nocivas que pueden perjudicar a la sociedad como el caso de muchos shooters en otros países, aunque esto puede ser corregido a tiempo si los padres hacen 
un fuerte trabajo al inculcar los valores morales dentro del seno familiar.

- La dinámica del transmedia permite que los prosumidores sean los que forjen los contenidos que estas comunidades consumen a través de plataformas digitales fortaleciendo su presencia con el apoyo de la comunicación virtual e incluso al permitir que en el plano real puedan desarrollar reuniones, eventos en bien de compartir momentos amenos.

- Se constató que la temática de juegos puede ser una oportunidad de monetizar, porque al convertirse en un gamer profesional o llegar a ser un líder de opinión las marcas relacionadas a este e-sport tratará de generar un patrocinio o mecenazgo con la finalidad de alcanzar este grupo de seguidores.

\section{REFERENCIAS BIBLIOGRÁFICAS}

Alonso-Geta, P. M. (2008). Protección de la infancia y nuevas tecnologías de la comunicación: el código PEGI de regulación de los videojuegos y juegos on-line. Teoría de la Educación. Educación y Cultura en la Sociedad de la Información, 9(3).

Ávila, J., \& Acosta, C. (2016). Epistemología del pensamiento visual contemporáneo desde el imaginario transdisciplinario. Anagramas, 14(28), 167-204.

Barthes, Roland (1977): Introducción al análisis estructural de los relatos. En El análisis estructural. Buenos Aires: Centro Editor de América Latina

Bertran, E., \& Chamarro, A. (2016). Videojugadores del League of Legends: El papel de la pasión en el uso abusivo y en el rendimiento. Adicciones, 28(1), 28-34.

Cano, L. C. (2017). El auge de nuevos modelos comunicativos: conectividad transmedia y conectividad tecnosocial. Estudio de caso: la Liga de optimistas pragmáticos. Index. comunicación: Revista científica en el ámbito de la Comunicación Aplicada, 7(1), 219-241.

Carrero, J. S. \& Pulido, P. C. (2012). DE CARA AL PROSUMIDOR: Producción y consumo empoderando a la ciudadanía 3.0. Revista ICONO14, 10(3), 62-84.

Charsky, D. (2010). From Edutainment to Serious Games: A Change in the Use of Game Characteristics. Games and Culture, 5(2), 177-198.

Costa Sánchez, C., \& Piñeiro Otero, T. (2012). Nuevas narrativas audiovisuales: multiplataforma, crossmedia y transmedia. El caso de Águila Roja (RTVE). Revista ICONO14, 10(2), 102-125.

Crisóstomo, R. (2016). Fannibals ministéricos: el poder del fandom. Index Comunicación, 6(2), 101-114.

Del-Moral-Pérez, M. E., \& Guzmán-Duque, A. P. (2017). Perfil de jugador virtual asociado a la motivación personal para jugar en CityVille. Revista de Educación a Distancia (RED), (54). https://doi.org/10.6018/red/54/3

Games, R. (2016). ¿Qué es League of Legends? Retrieved June 6, 2018, from https://euw.leagueoflegends.com/es/game-info/get-started/what-is-lol/

Guerrero-Pico, M. \& Scolari, C. A. (2016). Narrativas transmedia y contenidos generados por los usuarios: el caso de los crossovers. Cuadernos info, (38), 183-200. 
Hernández Ruiz, J. (2017). Diseñando una recepción participativa para universos transmedia: roles y desafíos. Tropelías, 28(21).

Herrero Diz, P., Lozano Delmar, J., Del Toro, A., \& Sánchez Martín, M. (2017). Estudio de las competencias digitales en el espectador fan español. Palabra Clave, 20(4), 947.

Hussain, Z., Williams, G. A., \& Griffiths, M. D. (2015). An exploratory study of the association between online gaming addiction and enjoyment motivations for playing massively multiplayer online role-playing games. Computers in Human Behavior, 50, 221-230.

INEC. (2018). Tecnologías de la Información y Comunicaciones (TIC'S) 2016. Retrieved June 5, 2018, from http://www.ecuadorencifras.gob.ec/documentos/web-inec/Estadisticas_Sociales/TIC/2016/170125.Presentacion_ Tics_2016.pdf

Kahn, A. S., Shen, C., Lu, L., Ratan, R. A., Coary, S., Hou, J., ... Williams, D. (2015). The Trojan Player Typology: A cross-genre, cross-cultural, behaviorally validated scale of video game play motivations. Computers in $\mathrm{Hu}$ man Behavior, 49, 354-361.

Miranda-Galbe, J. y Figuero-Espadas, J. (2016). El rol del prosumidor en la expansión narrativa transmedia de las historias de ficción en televisión: el caso de El Ministerio del Tiempo. Index Comunicación, 6(2), 115-134.

Nuangjumnonga, T., \& Mitomo, H. (2012). Leadership development through online gaming. In XIX Conferencia Bienal ITS, Bangkok 2012. Bangkok: ITS.

Rose, F. (2011). The Art of Immersion: How the Digital Generation Is Remaking Hollywood, Madison Avenue, and the Way We Tell Stories. W. W. Norton \& Company.

Scolari, C. A. (2013). Homo Videoludens 2.0: de Pacman a la gamification. Retrieved from https://repositori.upf.edu/handle/10230/26009

Scolari, C. A. \& Establés, M.-J. (2017). El ministerio transmedia: expansiones narrativas y culturas participativas. Palabra Clave, 20(4), 1041.

Scolari, C. (2017). El translector. Lectura y narrativas transmedia en la nueva ecología de la comunicación. La lectura en España. Informe 2017.

Sola Morales, S. (2015). Imaginarios sociales, procesos de identificación y comunicación mediática. Revista PRISMA.COM, 0(25). 